\title{
A Mechanism of Action for Hydroxychloroquine and Azithromycin to Inhibit Coronavirus Disease COVID-19
}

\author{
Charles D. Schaper, Ph.D. \\ Transfer Devices, Inc. \\ Union City, CA 94587 USA \\ text: $510-378-8260$ \\ Corresponding author: Charles D. Schaper, Ph.D. \\ cschaper@transferdevices.com
}

April 17, 2020

\begin{abstract}
Hydroxychloroquine and azithromycin have clinical promise to treat COVID-19, although its mechanism of action to inhibit the replication of coronavirus is unclear. Using molecular modeling and recent discoveries made by this lab on the structure of nucleic acids, a mechanism of action is developed for hydroxychloroquine (HCQ) and azithromycin (AZR) to inhibit the replication of the coronavirus disease COVID-19. The mechanism involves: (1) binding the $\mathrm{Cl}$ end-element of HCQ through ionic means to adjacent phosphate groups of the uracil nucleotide; (2) forming an intermolecular hydrogen bond of an NH group of HCQ to an open oxygen element of uracil; (3) binding $\mathrm{OH}$ end group of $\mathrm{HCQ}$ through ionic means with adjacent phosphate groups of the adenine nucleotide. The mechanism of action is extended to AZR as a drug delivery vector that collects $\mathrm{HCQ}$ and two ions of positive two charge, such as $\mathrm{Mg}^{2+}, \mathrm{Zn}^{2+}$ or $\mathrm{Ca}^{2+}$, and delivers the assembly to a secondary structure of single-strand RNA. As with HCQ, the structural biology of AZR is compatible for use as a collection and delivery vesicle including: (1) open access for the $\mathrm{Cl}$ end element and the $\mathrm{NH}$ group of HCQ to align and bind with Uracil, and (2) the ability to deliver and bind through ionic coupling of the $\mathrm{OH}$ end group of $\mathrm{HCQ}$ to the adenine nucleotide. The molecular ionic attachment of HCQ to RNA nucleotides enabled by AZR results in the inhibition of the replication capability of the coronavirus disease COVID-19.
\end{abstract}




\section{Highlights}

- Structural analysis of hydroxychloroquine indicates an excellent match for ionic coupling to the adenine - uracil RNA pairings of secondary structures of coronavirus to inhibit the translation and replication of COVID-19 virus;

- Structural analysis of azithromycin indicates binding pockets for ionic coupling of the end groups of hydroxychloroquine with significant clearance of the $\mathrm{Cl}$ and $\mathrm{NH}$ groups to permit coupling with the uracil nucleotide;

- A Mechanism of Action is developed for hydroxychloroquine used alone, without azithromycin;

- The activity of hydroxychloroquine to bind RNA nucleotides is compared and shown to be greater than that of chloroquine, thus identifying the importance for an $\mathrm{OH}$ end group;

- A Mechanism of Action is developed for hydroxychloroquine used in combination with azithromycin, which involves the assembly and delivery by azithromycin of hydroxychloroquine and two ionic coupling elements to adenine-uracil RNA pairings of the COVID-19 virus;

- The conclusions are compared against recent new results on the structural basis of DNA nucleotides as steroid molecules and its functional relation and coupling with steroid hormones;

- The atoms and groups comprising hydroxychloroquine including $\mathrm{Cl}, \mathrm{NH}$, aromatic rings, $\mathrm{OH}$, and a methyl group, as well as its overall dimension and positioning, are well suited for coupling to RNA nucleotides so as to inhibit the replication of the COVID 19 virus;

- Common ions of positive two charge including $\mathrm{Mg}^{2+}, \mathrm{Zn}^{2+}$, and $\mathrm{Ca}^{2+}$ are excellent coupling agents for binding hydroxychloroquine to adjacent RNA phosphate groups;

- The molecular description of the Mechanism of Action is applicable to optimize the structure of the coupling molecule, coupling agent, and delivery vector;

- Results are extensible to other single-strand and double-strand RNA viruses. 


\section{Introduction}

The coronavirus disease COVID-19 is presently spreading and causing infections with relatively high mortality $[1,2]$. As there is no vaccine for COVID-19 currently and as crossprotection is not provided from normative vaccines, most are susceptible to infection from SARS-CoV-2 $[3,4]$. The procedure to attenuate the impact of the disease is to implement mitigation strategies through social isolation thereby preserving health but devastating the economy. Therefore, a curative treatment is immediately needed as a stopgap to allow for time to study the disease and develop a long-term solution.

To treat COVID-19, initial clinical trials using the repurposed drugs hydroxychloroquine and azithromycin have shown promising results $[5,6,7,8]$. In fact, the President of the United States of America has negotiated to assemble a stockpile of hydroxychloroquine and azithromycin. Because both drugs are already approved by the FDA, if a treatment plan can be developed for these molecules, then a near-term solution is feasible. Accordingly, the drugs have been distributed and deployment has begun.

However, there is significant skepticism about the potential use of hydroxychloroquine, both within the mainstream media and the medical community $[9,10]$. The objections relate to the expectations on how long it will take to determine the efficacy of the drugs and whether significant side effects develop. Moreover, even though these drugs have received approval for use from the FDA, there is a lack of experience in applying the drugs for COVID-19 because it is a new coronavirus disease. For example, there is confusion as to whether hydroxychloroquine or chloroquine should be used. In addition, it is unclear as to the recommended dosing levels and the primary purpose of azithromycin.

These issues all relate to a lack of knowledge because a feasible mechanism of action for hydroxychloroquine and azithromycin to defeat the coronavirus disease COVID-19 has not been developed or even presented in modest detail. Moreover, it is difficult to comprehend that hydroxychloroquine, which is an anti-malarial agent, and azithromycin, an anti-biotic, would be useful against a single-strand RNA virus, and many debates cannot get beyond that issue in developing a reason to study these drugs for efficacy [11]. In terms of the research literature regarding a potential mechanism of action against the coronavirus disease COVID-19, there is speculation that the chlorine group of hydroxychloroquine, as with chloroquine, may interact with the phagolysosome [12]. A recent review article on the mechanisms of action of hydroxychloroquine and chloroquine notes that even for their intended use in the field of rheumatology, the mechanisms of action are still unknown and are classified as emerging, although pathways have been proposed suggesting that these drugs impact lysosomal activity, membrane stability, and alter transcription and signaling pathways, including alterations to cytokine production [13]. One in-silico study has indicated a mechanism of action involving interference of hydroxychloroquine with the ACE-2 receptor to prevent the coronavirus from entering the cell [14]. The need for a mechanism of action is reinforced by a recent study [15] that indicates that molecular mechanisms of action are in need of development for both hydroxychloroquine and chloroquine. More- 
over, there has been very little mention in these surveys about azithromycin either, other than the clinical trials mentioned above. Thus, detailed molecular models of a potential mechanism of action for hydroxychloroquine related to inhibition of replication has yet to be reported and elucidated. It is also the case that a mechanism of action is lacking for the molecular interactions and associated benefits of coordinating hydroxychloroquine (HCQ) and azithromycin (AZR).

In this article, applying results from recent discoveries that I have made on the structural basis of DNA $[16,17]$ and its functional interactions [16], a mechanism of action is developed for hydroxychloroquine and azithromycin to inhibit coronavirus disease COVID19. The results indicate an excellent structural configuration of the HCQ molecule in terms of its potential and capability to bind to the Adenine-Uracil nucleotides paired in a secondary structure of the single strand RNA, thereby inhibiting the further processing and replication of the coronavirus. Furthermore, the results indicate that the structural configuration of AZR is also excellent in terms of its capability to function as a receptor to first assemble two ions (for example, $\mathrm{Mg}^{2+}, \mathrm{Zn}^{2+}$, or $\mathrm{Ca}^{2+}$ ) along with $\mathrm{HCQ}$, and then deliver and bind together paired RNA strands of Adenine and Uracil bases arranged as a secondary structure of the single strand RNA coronavirus disease COVID-19. With this information of a detailed molecular mechanism of action for both HCQ and AZR, it is possible to develop effective treatment plans to inhibit the coronavirus disease COVID-19.

\section{Results}

To understand and develop this mechanism of action of hydroxychloroquine and azithromycin to inhibit coronavirus COVID-19, and more generally single strand RNA nucleotide viruses, it is first necessary to describe fundamental results originally developed by this lab on the mechanism of action of binding steroid hormones to DNA nucleotides, which is described in Section 2.1. After the discussion on DNA nucleotides, a mechanism of action is then developed in Section 2.2 for the interaction of hydroxychloroquine and the RNA nucleotides of the coronavirus, without the use of azithromycin, since it has been noted that there is efficacy with the use of hydroxychloroquine alone. Next, in Section 2.3 a mechanism of action is developed for hydroxychloroquine in combination with azithromycin for the inhibition of the coronavirus disease COVID-19.

\subsection{Receptor Mediated Binding of Steroid Hormones to DNA Nucleotides}

In a series of preprints $[16,17]$, striking results were presented which indicated a remarkable finding that showed for the first time that the base pairings of the four DNA nucleotides perfectly form the four ring structure of a steroid molecule. Furthermore, critical to a basic understanding of DNA and as will be seen to an understanding of a mechanism of action of hydroxychloroquine, this finding led to a resolution of a mystery as to why the adeninethymine pairing has only two internal hydrogen whereas cytosine-guanine has three internal 
hydrogen bonds. It was shown in my referenced preprints, and indicated in Figure 1, that the third hydrogen bond for A-T and T-A is formed when the A-T and T-A nucleotides are coupled with corticosteroids, such as cortisol, which has an oxygen functional group that is perfectly positioned to form a hydrogen bond with the accessible oxygen-based functional group of thymine. Apparently, this is critical, as it can be evaluated in terms of comparing the activity of the synthetic corticosteroids prednisone and prednisolone, in which the only difference is the hydroxyl group at the mid-molecule location, but the activity of prednisone is absent, whereas prednisolone is five times more powerful than cortisol.

The structural biology of this binding interaction is presented in Figures 1(a) and (b), which uses the representative steroid hormones cortisol and testosterone in a manner such that each of the four nucleotide pairings are related to a steroid hormone, such that three internal hydrogen bonds are formed. Since the C-G and G-C pairing already have three hydrogen bonds, testosterone is paired, and since A-T and T-A, cortisol is paired since the mid-sectional functional group must couple with the available group of Thymine. It is noted that in order to get the structural match for each of the pairings, the T-A and $\mathrm{C}-\mathrm{G}$ pairings were required to be matched on the "back" of the molecule to its normal presentation. Effectively this forms a structural code to DNA as expressed in Figure 1(c).

While the structural match is intriguing, the functional nature of the interaction also must be defined, and that I did as well, as first described in the preprint [16]. To facilitate this binding process, it is first necessary to consider the interaction of the steroid hormone with its receptor. Here, we will evaluate the steroid hormone cortisol and its association with the glucocorticoid receptor. The conventional thinking on receptors of steroid hormones was that hydrogen bonding was only deployed, but I showed that the receptor is also capable of holding two ions that possess a charge of positive two each, as shown in Figure 2(a) for cortisol and representative residues of the ligand binding domain of the glucocorticoid receptor and two calcium ions [18]. In addition to stabilizing the complex, the ions provide an electrostatic potential with two separated positive charges that thereby provide directionality and alignment capability, as indicated in Figure 2(b). This stability and directionality enable the receptor complex to mobilize.

After association with $\mathrm{Ca}^{2+}$ ions, the steroid hormone-receptor-ion complex is ready to mobilize with interaction with the DNA molecule. After passage into the nucleoplasm, the steroid hormone- receptor-ion complex aligns through electrostatic interaction with the DNA molecule using the phosphate groups of adjacent and paired nucleotides as the alignment mechanism. For cortisol, and according to Figure 1(a) and (b), it is also necessary that this alignment and interaction also involve the A-T or T-A pairing, since an available group of Thymine is required to achieve three internal hydrogen bonds. Upon transfer of the two ions and the steroid hormone to the DNA nucleotide pairs, a stable structure results as indicated in Figure 3(a) in which the interaction of cortisol with $\mathrm{Ca}^{2+}$ ions and with the $\mathrm{PO}_{4}^{-}$ions of the A-T base pairing, thus forming a strong ionic bond. The calcium ions are stabilized at approximately the mid-way point of adjacent phosphate groups and the lateral separation. In Figure 3(b) the electrostatic potential map is indicated of the 


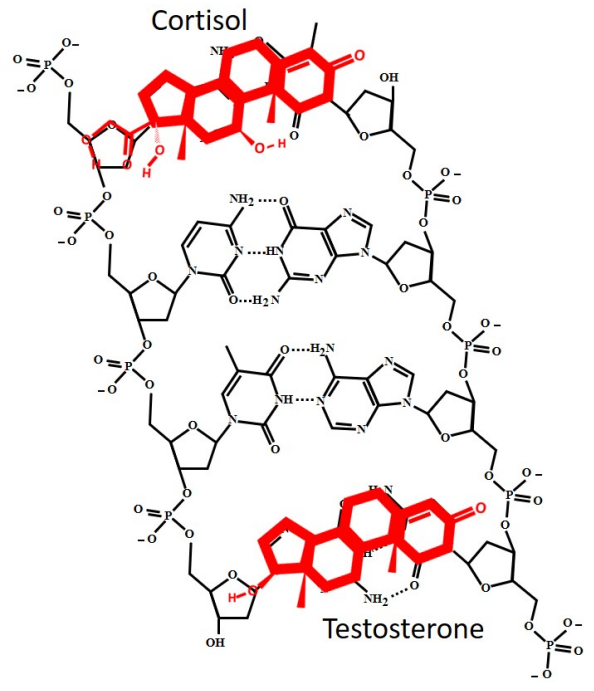

(a) Front Structural Symmetry

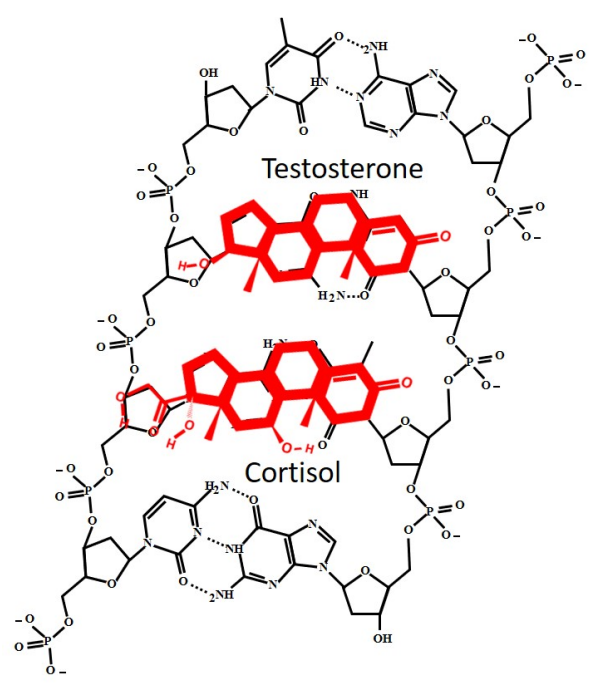

(b) Back Structural Symmetry

\begin{tabular}{|c|c|c|}
\hline $\begin{array}{c}\text { Base } \\
\text { Pair }\end{array}$ & $\begin{array}{c}\text { Steroid } \\
\text { Orientation }\end{array}$ & $\begin{array}{c}\text { Steroid } \\
\text { Class }\end{array}$ \\
\hline $\mathrm{A}-\mathrm{T}$ & $\mathcal{F}$ & $\mathbb{H}$ \\
$\mathrm{T}-\mathrm{A}$ & $\mathcal{B}$ & $\mathbb{H}$ \\
$\mathrm{C}-\mathrm{G}$ & $\mathcal{B}$ & $\mathbb{S}$ \\
$\mathrm{G}-\mathrm{C}$ & $\mathcal{F}$ & $\mathbb{S}$ \\
\hline
\end{tabular}

(c) New Code

Figure 1: This work was first described in my preprints [16, 17], which indicates that the structural basis of DNA is the steroid molecule, and that there is a correspondence cortisol-like steroid hormone with the $A-T$ and $T$-A pairs and the testosterone-like steroid hormones with the $C-G$ and $G$ - $C$ pairs. This association is established to produce three internal hydrogen bonds for each of the DNA nucleotide arrangements as the mid-molecule hydroxyl group of cortisol-like molecules forms a hydrogen bond with the functional group of Thymine. To achieve the configuration for each of the four pairs, there is a (a) front-side and (b) back-side orientation in order to get the three hydrogen bonds, and to have the proper orientation. (c) These developments thus have a new code for DNA nucleotide sequences, comprised of the spatial relation of the steroid hormone to the DNA molecule on the front $\mathcal{F}$ or the back $\mathcal{B}$, and to the class of steroid hormone, either cortisol-like $\mathbb{H}$, with a mid-molecule functional group, or testosterone-like, $\mathbb{S}$. The structural match between DNA nucleotides and steroid hormones is perfect and undeniable.

combined structure, and it is noted that there is a charge normalization in the middle with the retention of a smaller positive charge at the calcium ions. A significant stabilization of the DNA molecule results of $5,996 \mathrm{~kJ} / \mathrm{mol}$ [16], which can be distributed to induce gene 


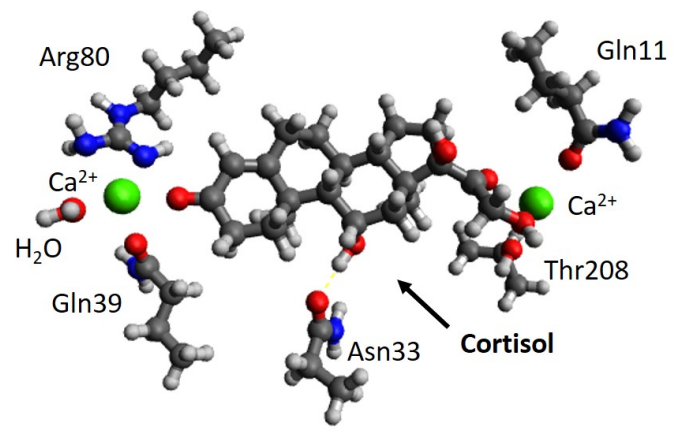

(a) Cortisol-Ca ${ }^{2+}-\mathrm{GR}$

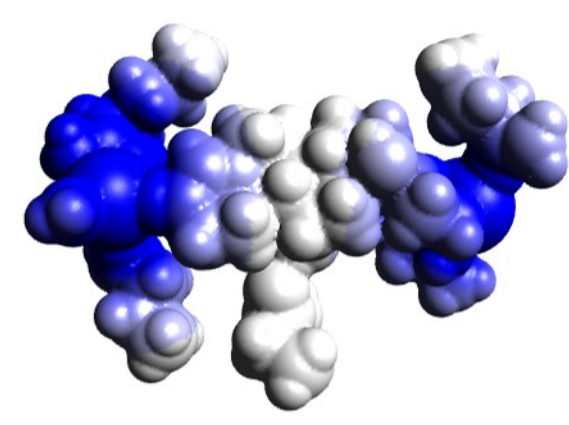

(b) Cortisol- $\mathrm{Ca}^{2+}-\mathrm{GR}$ electrostatic

Figure 2: For a representation of the ligand binding domain of a glucocorticoid receptor, (a) the configuration with a combination of hydrogen bonding and coupling to calcium ions (shown in green); (b) The electrostatic potential of the complex indicating two separate regions of positive charge.

transcription. However, for the analysis of RNA strands, and the coronavirus disease, this stabilization will be used to inhibit the RNA molecule from translation, as will be studied in the next section.

The calculations and developments for the steroid hormone-DNA interaction were performed for the calcium ion, but the results hold for other ions, particular $\mathrm{Mg}^{2+}$ and $\mathrm{Zn}^{2+}$. The point is to have significant positive charge to permit interaction with adjacent oxygen groups on the phosphate ions of the nucleotides. Both $\mathrm{Mg}^{2+}$ and $\mathrm{Zn}^{2+}$ are utilized in our upcoming study on RNA to indicate the approach in a broader perspective for ionic elements and others, such as $\mathrm{Mn}^{2+}$, capable of a positive two charge.

\subsection{Mechanism of Action for Only Hydroxychloroquine (without Azithromycin)}

Now, a mechanism of action will be developed for the use of hydroxychloroquine (HCQ) alone, that is without the use of azithromycin, as it has been reported to work to resolve the coronavirus disease COVID-19. In addition, as the use of chloroquine (CLQ) alone has also been reported to have some efficacy albeit less than HCQ, that will be discussed as well. To develop these results, I will apply my discoveries on: (1) the structural biology of the steroid hormone in its perfect relationship with the structural form of nucleic acids, (2) the ionic-phosphate-DNA coupling mechanism, of either $\mathrm{Ca}^{2+}, \mathrm{Mg}^{2+}$, or $\mathrm{Zn}^{2+}$ to adjacent $\mathrm{PO}_{4}^{-}$ groups; (3) the receptor mediated delivery connection and delivery mechanism. It is critical that the mechanism of action for hydroxychloroquine be developed through molecular modeling, such that the purpose of each element comprising the drug can be defined, so as to deploy the material effectively, and to optimize the approach.

A major result is indicated in Figure 4, which relates for the first time the HCQ molecule 


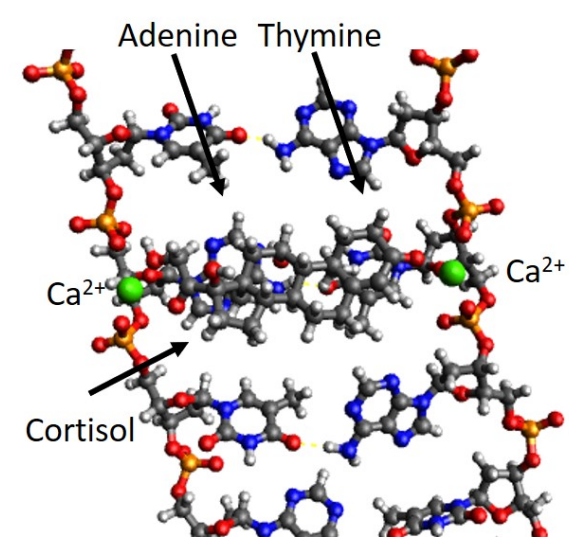

(a) Cortisol- $\mathrm{Ca}^{2+}$-Adenine-Thymine

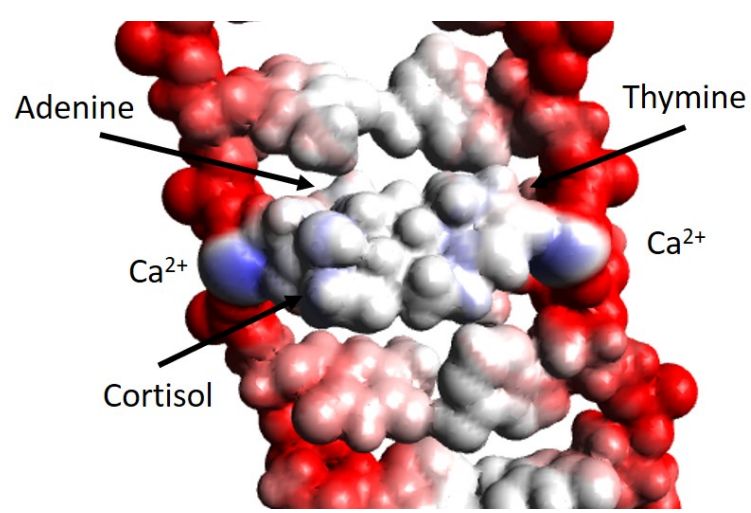

(b) Electrostatic Potential

Figure 3: The glucocorticoid receptor containing the ions and steroid hormone, calcium and cortisol in this case, are aligned through electrostatic forces and transferred to the A-T nucleotide pairing in a configuration so as to achieve a hydrogen bond with Thymine. after integration of the cortisol molecule with calcium ions, and release from the ligand binding domain, the interaction with the Adenine-Thymine pairing is shown in (a). It is noted that the calcium ions have stabilized at the middle of the adjacent phosphate groups (shown in orange) and the lateral separation is like what was present with the ligand binding domain prior to integration. In (b) the electrostatic potential map is indicated of the combined structure in which a small positive charge from the calcium ions is present.

to the adenine-uracil pairing of double-strand RNA. As seen, the structural biology of hydroxychloroquine is excellent for binding directly to the pairing of the Adenine-Uracil nucleotides. It has a certain equivalency to the cortisol binding to the A-T pairing of DNA as shown in Figure 1(a) and 3(a). First, it is emphasized here that the molecule HCQ has the right dimensions to extend between the two strands of RNA. Second, and most importantly, HCQ can achieve a hydrogen bond with the unmatched functional group of Uracil, which I have indicated above is critical in order for a binding to happen in discussion of the cortisol - thymine relation. Third, the $\mathrm{OH}$ end-group and $\mathrm{Cl}$ end-element are available to bind to the phosphate backbone, much as the case with steroid hormones and DNA interaction of Figure 1(a) and (b). Further, the two aromatic rings of HCQ align to two of the four effective rings comprising the A-U pairing and the side chain of HCQ is perfectly positioned.

As the structural match between HCQ and the A-U pairing of RNA nucleotides is excellent, it is necessary to describe a binding mechanism. To do this, in Figure 5, the relationship between the glucocorticoid receptor (GR) is shown, in which the cortisol steroid hormone in Figure 2 is replaced with HCQ, and here we use $\mathrm{Mg}^{2+}$ as the coupling agent. It indicates that the GR can retain HCQ, which should not be surprising since the structural equivalency of HCQ to cortisol was described earlier. Moreover, my work on showing 


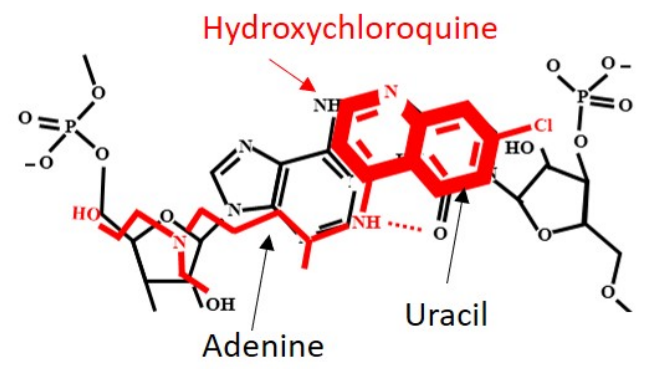

Figure 4: The structural biology of hydroxychloroquine (HCQ) is excellent for binding directly to the pairing of the Adenine-Uracil RNA nucleotides. The molecule HCQ has the right dimensions, as the $\mathrm{OH}$ end group and the $\mathrm{Cl}$ end element can bind to the adjacent phosphate groups on the respective RNA strand. Most importantly, HCQ, through the NH group on the chain extending from the aromatic rings, which is perfectly positioned, can achieve a hydrogen bond with the unmatched functional group of Uracil, which is very important analogous to the hydrogen bond produced by cortisol coupling to Thymine in DNA nucleotides. Furthermore, the two aromatic rings of $H C Q$ align to two of the four effective rings comprising the Adenine-Uracil pairing.

prostaglandin E2 as a contaminant to the GR also indicates that there is competition for the ligand binding domain of the GR [18]. The relation between HCQ and the GR also shows that there is a hydrogen bond at the $\mathrm{NH}$ group and the corresponding amino acid of the LBD of GR, which is Asn33 separated by $1.852 \AA$. Thus, after incorporation of HCQ with the GR, and incorporation of two ions, it is transported to the double strands of the RNA within the cytoplasm and delivery of HCQ in a manner like that of cortisol, as indicated in Figure 3(a).

Thus, the mechanism of action of HCQ alone, acting without the aid of azithromycin, to inhibit coronavirus COVID-19 is to interact with the GR, with delivery of HCQ and the associated ionic pair, to the A-U pairing associated with a secondary structure of the single RNA strand of the coronavirus disease COVID-19. The secondary structures occur frequently for single strand RNA, when the strand doubles back on itself to form a double stranded pair. This result is indicated in Figure 6(a) for the A-U pairing, $\mathrm{Mg}^{2+} / \mathrm{PO}_{4}^{-}$ coupling. In proximity to the $\mathrm{NH}$ group, here is a methyl group which acts as a spacing element and will lead its interaction with the A-U nucleotide pairing, in a manner similar to cortisol. It can also be seen that the hydrogen bond distance between the $\mathrm{NH}$ group of HCQ and the O element on Uracil is $4.120 \AA$, which is similar to that which is obtained for cortisol-thymine with this software package. The distance between the chlorine element and the $\mathrm{Mg}^{2+}$ coupling element is $2.954 \AA$, which is consistent with modeling of cortisol coupling to DNA nucleotides. The distance between the $\mathrm{OH}$ end group of HCQ to $\mathrm{Mg}^{2+}$ is $2.088 \AA$. The electrostatic potential is presented in Figure 6(b), which shows that a small positive charge remains on $\mathrm{Mg}^{2+}$ for further interaction. 


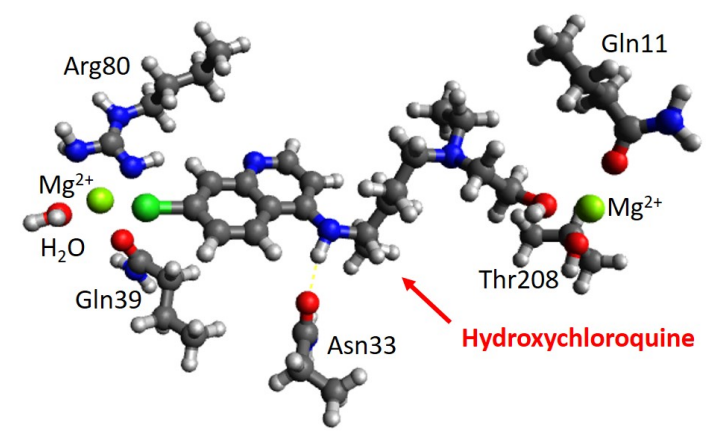

Figure 5: Without another receptor choice, HCQ still shows favorable interaction with the glucocorticoid receptor, as the match is also excellent as each functional group is coupled, including tight hydrogen bond of the nitrogen group which will ultimately bind with the available functional group of Uracil. This result can be compared with the occupation of cortisol with the glucocorticoid receptor.

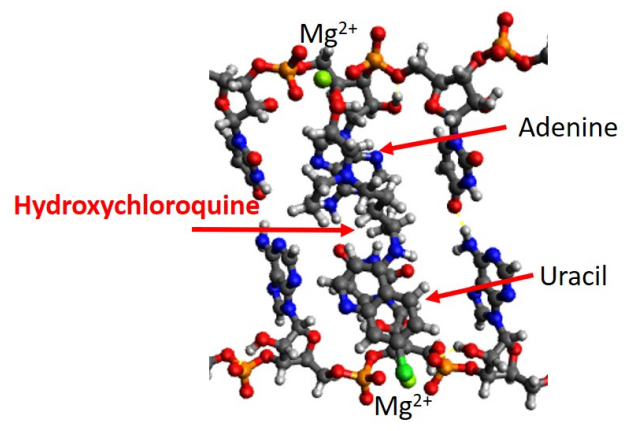

(a) $\mathrm{HCQ}-\mathrm{Mg}^{2+}-\mathrm{RNA}$ secondary structure

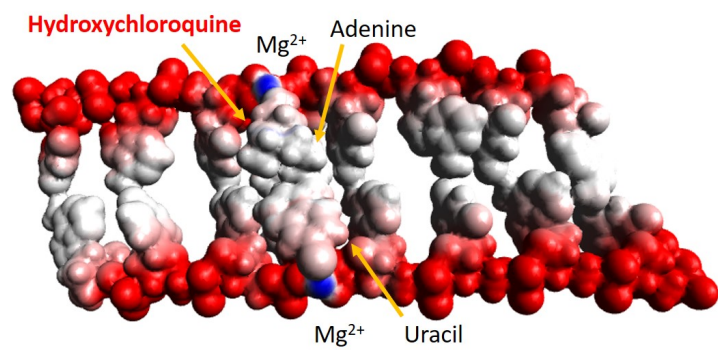

(b) $\mathrm{HCQ}-\mathrm{Mg}^{2+}-\mathrm{RNA}$ secondary electrostatic

Figure 6: In defining the mechanism of action for Hydroxychloroquine Alone to Inhibit Coronavirus Disease COVID-19 is shown: (a) the binding of HCQ to the A-U RNA of the coronavirus $R N A$ sequence in a folded secondary structure. (b) the electrostatic potential including the $\mathrm{Mg}^{2+}$ is still available as differentiated, and thus can be used for additional binding. The overall complex will preclude SARS-CoV-2 from translation, and thus replication, and effectively inhibit the coronavirus disease COVID-19.

In Figure 7, the interaction with $\mathrm{Zn}^{2+}$ as a coupling agent is indicated, and approximately equivalent results are obtained, and thus either $\mathrm{Mg}^{2+}, \mathrm{Ca}^{2+}$, or $\mathrm{Zn}^{2+}$ is sufficient to achieve this coupling. Finally, in terms of CLQ to inhibit coronavirus disease COVID19, it is possible, but not as effective as HCQ, because CLQ does not have an OH end group for coupling with the double-stranded secondary structure of the single-strand RNA coronavirus. However, there is an NR3 group that can bind with the second strand, and in any event the $\mathrm{Cl}$ and $\mathrm{NH}$ groups can bound with the single strand, which itself should 
be helpful in reducing the capability for translation and replication.

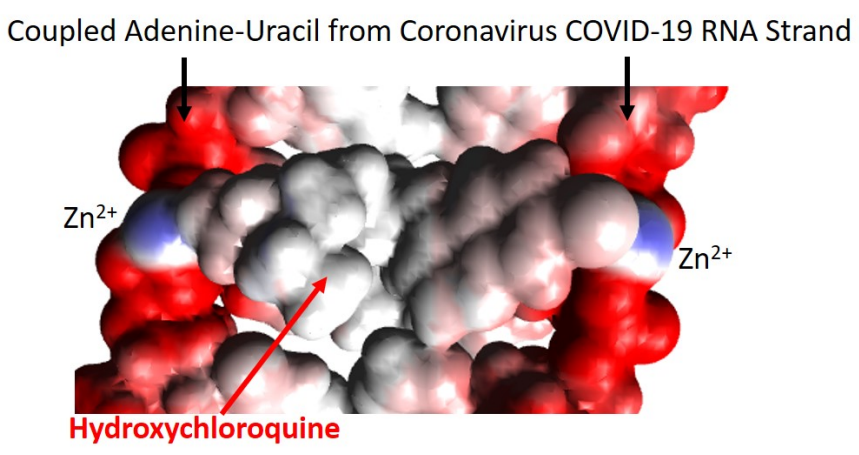

Figure 7: Shown here is $\mathrm{Zn}^{2+}$ coupling hydroxychloroquine to paired RNA strands in a secondary structure of single RNA strand coronavirus COVID-19. The mechanisms of action are applicable to ionic coupling points with a positive two charge, and include $\mathrm{Zn}^{2+}, \mathrm{Ca}^{2+}$, and $\mathrm{Mg}^{2+}$. The key point is that the coupling point be able to interact with the phosphate groups from adjacent nucleotides, and interact with the drug, in this case hydroxychloroquine, steroid hormone or steroid molecule.

\subsection{Mechanism of Action for Hydroxychloroquine and Azithromycin}

In a manner similar to that with which steroid hormones interact with receptors, in order for hydroxychloroquine to achieve inhibition by binding directly onto paired RNA strands of coronavirus, it is first necessary to associate HCQ with the coupling elements of a receptor that is more effective than the glucocorticoid receptor, which nominally contains cortisol.

It is in the capacity of a receptor that Azithromycin (AZR) is crucial. My analysis indicates that AZR is particularly competent at achieving an excellent binding pocket for hydroxychloroquine with the ionic coupling agents of $\mathrm{Mg}^{2+}, \mathrm{Ca}^{2+}$, or $\mathrm{Zn}^{2+}$. In Figure 8(a) two positively charged ions $\mathrm{Mg}^{2+}$ are arranged at two separated points within the Azithromycin structure, which will present a targeted binding site. In Figure 8(b) Hydroxychloroquine binds to the structure to form a complex comprised of Hydroxychloroquine $\mathrm{Mg}^{2+}$ - Azithromycin, which is stabilized and ready for transport. The distance between the two $\mathrm{Mg}^{2+}$ is $11.445 \AA$, which is less than that of the glucocorticoid receptor, which is $18.410 \AA$. This will alter the presentation of the HCQ to Uracil. Also, note that the positioning of the chlorine end-element as well as the interior NH group HCQ is available for binding to Uracil, as it is not hindered. The two aromatic rings of HCQ also provide structure, and there is a spacing element, the methyl group, and the hydrogen element of the $\mathrm{NH}$ group on the side chain is available for hydrogen bonding, which is analogous to cortisol. Thus, the likelihood for binding HCQ wto the A-U pairing is excellent with the use of azithromycin as the receptor. 


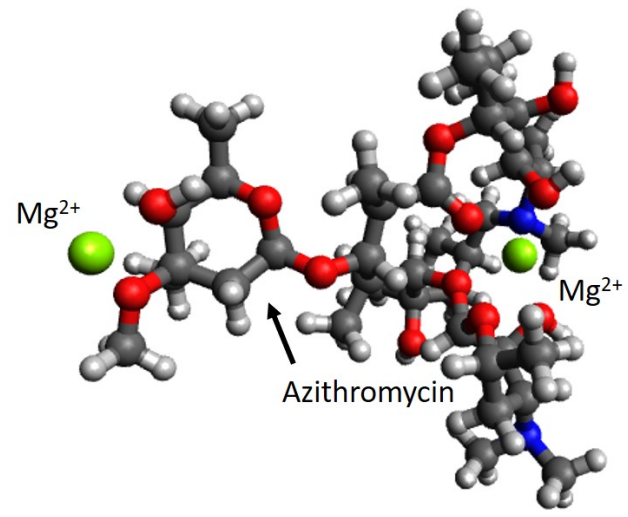

(a) Azithromycin- $\mathrm{Mg}^{2+}$

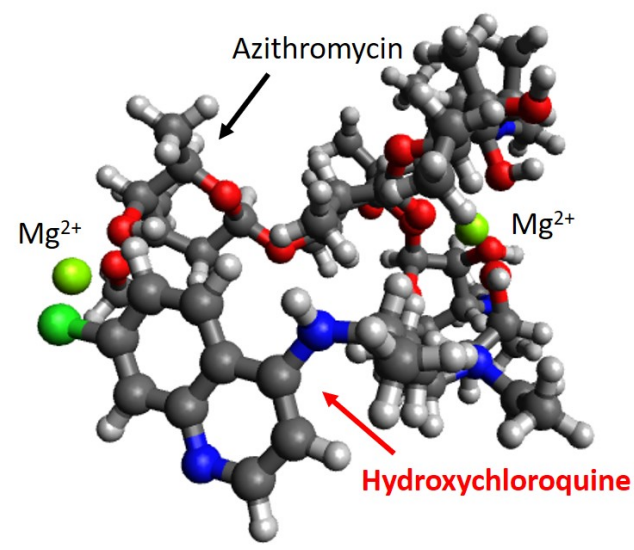

(b) Azithromycin- $\mathrm{Mg}^{2+}-$ Hydroxychloroquine

Figure 8: Before interaction with the RNA strands of the coronavirus disease COVID-19, it is first necessary to assemble the drug with the ion coupling elements. In (a) two $\mathrm{Mg}^{2+}$ are arranged at two separated points within the Azithromycin structure. Then in (b) Hydroxychloroquine binds to the structure to form a complex comprised of Hydroxychloroquine $\mathrm{Mg}^{2+}$ - Azithromycin, which is stabilized and ready for transport. Note the positioning of $H C Q$ is available for binding, as it is not hindered, especially the chlorine group (colored in darker green). The two aromatic rings of $H C Q$ also provide structure, and there is a spacing element, the methyl group, and the hydrogen element connected to the nitrogen element on the side chain is available for connection. Thus the likelihood for connection with the A-U pairing is excellent.

In evaluating the energy associated with the complex, for Azithromycin alone, the energy level is $1213.7 \mathrm{~kJ} / \mathrm{mol}$; for Azithromycin $-\mathrm{Mg}^{2+}$, the energy is $298.2 \mathrm{~kJ} / \mathrm{mol}$, and for and for the complete complex Azithromycin $-\mathrm{Mg}^{2+}-$ Hydroxychloroquine is 249.6 $\mathrm{kJ} / \mathrm{mol}$, which is a relative energy level associated with the disassociation requirements. The energy levels thus indicate significant stabilizing forces with the incorporation of magnesium ions. In addition, the incorporation of Hydroxychloroquine moderately stabilizes the overall complex, which will permit the unit to hold together during the transport process, and favor its association to begin the process. It is noted that the energy level with $\mathrm{Zn}^{2+}$ is $364.54 \mathrm{~kJ} / \mathrm{mol}$ and for $\mathrm{Ca}$ is $589.74 \mathrm{~kJ} / \mathrm{mol}$, thus in terms of stability magnesium is favored, followed by zinc, then calcium.

The electrostatic potential is the driving force to transport the HCQ-Ion-AZR complex to the negatively charged intracellular RNA strand. In Figure 9(a), it is noted that the AZR receptor enables separation of positive charges, although the charge associated with the chlorine molecule is directly exposed and strong. While the coronavirus disease COVID-19 is a single-strand of RNA, there are secondary structures, in which the RNA strand loops back across a number of regions to produce a complementary base pairing. In Figure 9(b) 
the electrostatic potential map is indicated for an aligned association wth the HCQ-IonAZR complex to the RNA nucleotides as a secondary structure of the coronavirus disease COVID-19 configured as AUAUAUAU.

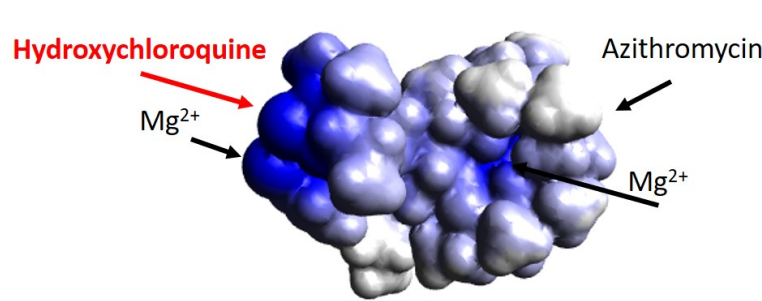

(a) Azithromycin- $\mathrm{Mg}^{2+}$-Hydroxychloroquine

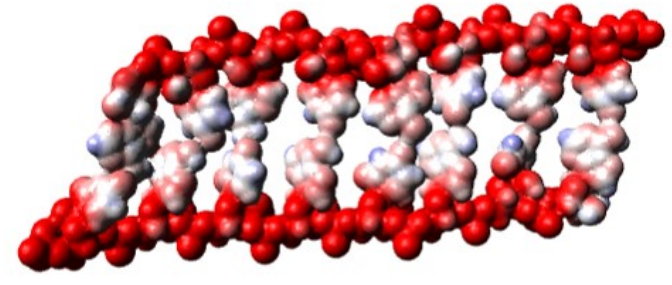

(b) Adenine-Uracil pairing electrostatics

Figure 9: Electrostatic potential is the driving force to transport the complex to the RNA strands comprising the coronavirus disease. In (a), the electrostatic potential is indicated for the Hydroxychloroquine- $\mathrm{Mg}^{2+}$-Azithromycin complex, where it is noted that there are two separate positive charges, although the charge associated with the chlorine molecule is exposed and strong. In (b) the electrostatic potential map is indicated for the target point, which is the RNA nucleotides of the coronavirus disease COVID-19 configured in a secondary structure.

In the initial presentation to the uracil nucleotide, the chlorine element of hydroxychloroquine is connected through the $\mathrm{Mg}^{2+}$ ion to the adjacent phosphates associated with Uracil. After the coupling, the connection point to Azithromycin begins to pull away as indicated in Figure 10 which is the top-down view indicating that the molecule is focused on the uracil side of the secondary structure of the RNA, and at this point only covers approximately half of the RNA pair. In Figure 10(b), the side view indicates the motion of the elements as the transfer of the ion occurs while stabilizing the chlorine element, in which it is noted that there is no steric hinderance which would prevent the binding. A critical point is that hydrogen bond coupling is achieved through the NH group from the side chain of the double aromatic structure of HCQ, which in this model and configuration during the transfer process is $3.773 \AA$, consistent with the measurement of the cortisolthymine binding. As indicated previously, hydrogen bond coupling with thymine, or uracil in this case, is enabling as evidenced by the negligible activity of prednisone relative to prednisolone.

After achieving the initial presentation of the molecule at the chlorine side of the HCQ molecule, and the transfer of the coupling ion from the receptor to the phosphate group, the AZR receptor is then free to rotate away as indicated in Figure 11(a), and thus the AZR receptor, which contains an ion with positve charge two, will motion toward the other side of the RNA double strand secondary structure, that is the Adenine nucleotide. In Figure 11(b), the binding of the hydroxyl group to the Adenine complex is indicated in 


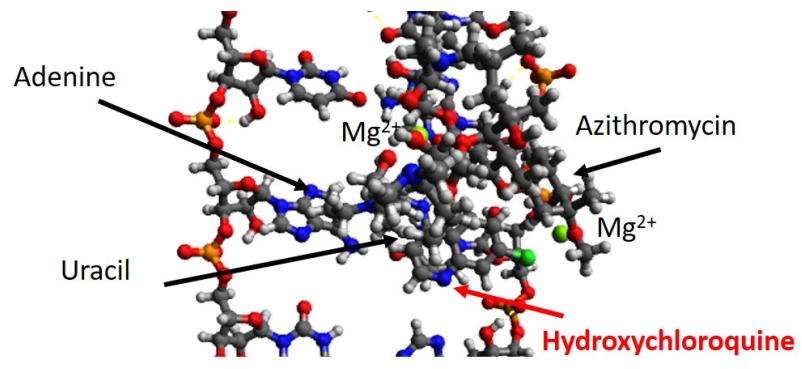

(a) Initial Presentation - Top Down View

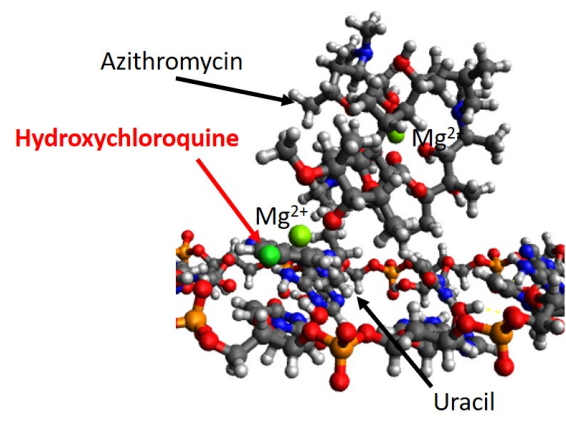

(b) Initial Presentation - Side View

Figure 10: The initial presentation of the complex to the uracil nucleotide in which the binding of the chlorine element of hydroxychloroquine through the $\mathrm{Mg}^{2+}$ ion transfer from the complex to the phosphate adjacent pairing, in which Azithromycin begins to pull away (a) the top-down view indicates that the molecule is focused on the uracil side of the secondary structure of the RNA. (b) The side view indicates the motion of the elements as the transfer of the ion occurs while stabilizing the chlorine element, in which it is noted that there is no steric hinderance which would prevent the binding from resulting.

which the second $\mathrm{Mg}^{2+}$ ion is transferred to the phosphate group. This then completes the coupling of the HCQ molecule to an A-U pairing of the genetic material associated with the coronavirus disease.

After completing the coupling of HCQ to the double strand of RNA, bound together through the ionic element, the Azithromycin molecule can then diffuse away from the complex, as indicated in Figure 12, since Azithoromycin has a relative neutral charge and is not attracted to the phosphate groups of the RNA strands. The coupling will have the effect of locally stabilizing the double strand, and additional coupling points will further inhibit the further actions of the coronavirus disease genetic material to translate and thus will suppress replication. In Figure 12(b) The electrostatic potential of the integration is shown which indicates that there is positive charge that remains, which could be used for further developments and attachments to promote its removal.

In Table 1, the steps are outlined for the use HCQ and AZR to inhibit the coronavirus disease COVID-19. An overview of the approach involves the coupling of HCQ and AZR through an ionic element, which can be for example $\mathrm{Mg}^{2+}, \mathrm{Zn}^{2+}$, or $\mathrm{Ca}^{2+}$. The energy levels were best expressed for $\mathrm{Mg}^{2+}$, which was slightly better than $\mathrm{Zn}^{2+}$. After ionic coupling, the HCQ molecule is very well positioned for interaction with an RNA nucleotide, Uracil, of COVID-19, arranged in a secondary structure assembled with Adenine. This nucleotide is favored because there a structural symmetry with the two rings of HCQ, and the nitrogen group on the side chain is well positioned to achieve hydrogen bonding with Uracil. After securing this connection, the end of the receptor AZR that was previously attached, is 


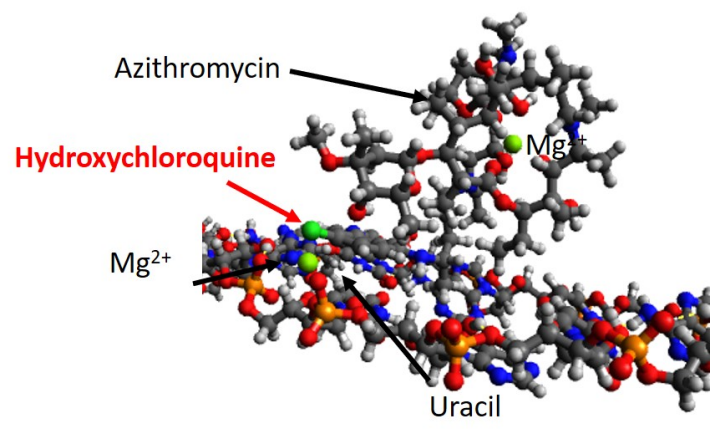

(a) Uracil-Hydroxychloroquine Binding

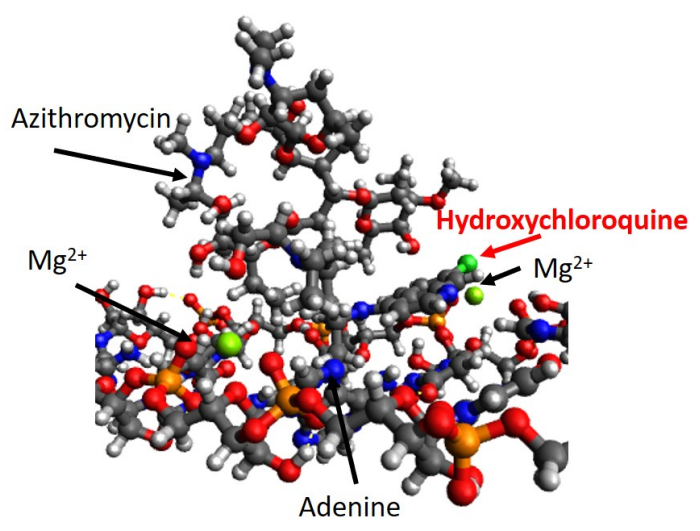

(b) Adenine-Hydroxychloroquine Binding

Figure 11: In (a), the binding of the chlorine group to the phosphate chain at the uracil site, and the binding of the hydrogen bonding to the unpaired functional group of Uracil. In addition, without the magnesium ion available, the Azithromycin molecule rotates away from the Uracil molecule and towards the Adenine side. In (b), the binding of the hydroxyl group to the Adenine complex is indicated in which the second $M g^{2}+$ ion is transferred to the phosphate group.

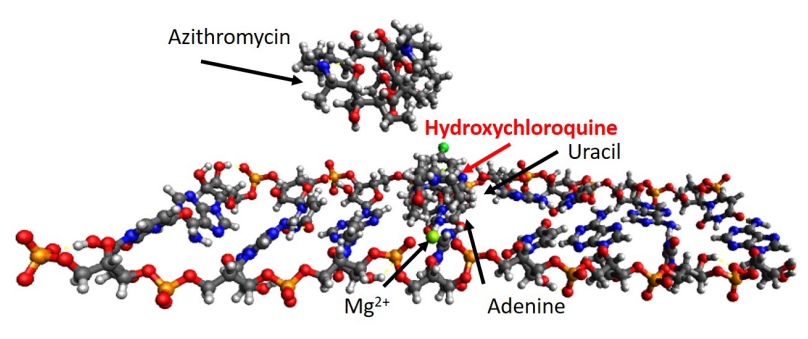

(a) Hydroxychloroquine- $\mathrm{Mg}^{2+}-\mathrm{RNA}$ integration

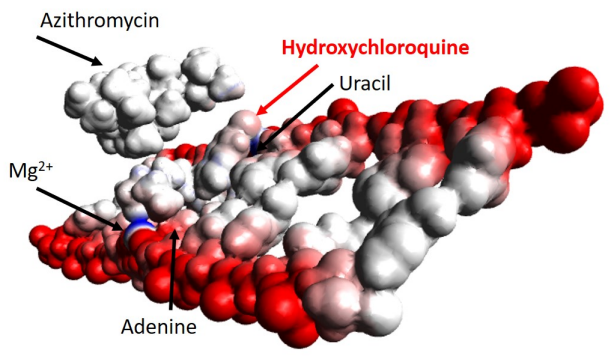

(b) Hydroxychloroquinine- $\mathrm{Mg}^{2+}$-RNA electrostatics

Figure 12: (a) After the transfer of Hydroxychloroquine to the RNA strands, the Azithromycin diffuses away from the complex. (b) The electrostatic potential of the integration, indicating that there is positive charge that remains, which could be used for further developments. The Azithromycin molecule is neutral and is thus not attracted to the RNA strands.

released which enables a rotation of the HCQ molecule containing the second ionic element to bind to the adjacent phosphate groups associated with the Adenine nucleotide. The mechanism of transferring the ionic element is conducted and stabilized the HCQ molecule. AZR is then diffused away from the HCQ-AZR-RNA complex. The coupling will inhibit the 
molecule from further translation and replication, and thus suppress the disease. Additional binding interactions will promote improved inhibition.

\begin{tabular}{|l|l|}
\hline Step & Function \\
\hline 1 & $\begin{array}{l}\text { Entry by diffusion of Hydroxychloroquine and Azithromycin into the cell } \\
\text { infected with coronavirus disease COVID-19 }\end{array}$ \\
\hline 2 & $\begin{array}{l}\text { Association of two ion elements at lateral spaced binding pockets of } \\
\text { Azithromycin. (In the steps that follow, } \mathrm{Mg}^{2+} \text { will be used as the linking } \\
\text { element, noting that } \mathrm{Ca}^{2+} \text { and } \mathrm{Zn}^{2+} \text { would also work well.) }\end{array}$ \\
\hline 3 & $\begin{array}{l}\text { Binding of Hydroxychloroquine to Azithromycin though coupling to } \\
\mathrm{Mg}^{2+} \text { ions }\end{array}$ \\
\hline 5 & $\begin{array}{l}\text { Transport through electrostatic attraction to align to an A-U secondary } \\
\text { structure pairing of the single coronavirus RNA strand }\end{array}$ \\
\hline 5 & $\begin{array}{l}\text { Binding of the chlorine element of Hydroxychloroquine to a Mg }{ }^{2+} / \mathrm{PO}_{4}^{-} \\
\text {lease of the first site of Azithromycin coupling to Mg }\end{array}$ \\
\hline 8 & $\begin{array}{l}\text { Rotation of remaining complex of Mg } \\
\text { Hydroxychloroquine, to the Adenine side of the A-U RNA coronavirus } \\
\text { secondary pair }\end{array}$ \\
\hline 9 & $\begin{array}{l}\text { Transfer of Mg }{ }^{2+} \text { to the phosphate group of the Adenine nucleotide } \\
\text { complex with hydroxy group of Hydroxychloroquine remaining bound }\end{array}$ \\
\hline $\begin{array}{l}\text { Diffusion of Azithromycin away from the Hydroxychloroquine-Adenine- } \\
\text { Uracil-Mg }\end{array}$ \\
\hline 10 & $\begin{array}{l}\text { Inhibition of translation and replication of the coronavirus disease } \\
\text { COVID-19 with cytoplasmic defeat through degradation via intracel- } \\
\text { lular lysosome }\end{array}$ \\
\hline
\end{tabular}

Table 1: The main steps in the association of the Hydroxychloroquine and the Azithromycin molecules to combat the coronavirus disease COVID-19.

\section{Discussion}

A structural biology analysis is in excellent agreement with the hypothesis put forth in this research article of inhibiting the coronavirus disease COVID-19 by the attachment of hydroxychloroquine (HCQ) to pairings of uracil nucleotides and adenine nucleotides. HCQ has three functional groups well positioned and of sufficient function to interact with the adenine-uracil nucleotide pairing, as follows: (1) the Cl end element of HCQ is positioned at the exact location of the aromatic ring in correspondence to the steroid hormone, cortisol, which permits its interaction as a nucleophile with the phosphate backbone through ionic coupling; (2) the NH group of HCQ is positioned at a precise location which permits 
hydrogen bonding with the available functional group of Uracil, in correspondence with that of the steroid hormone, cortisol, which apparently is critical and necessary; (3) the $\mathrm{OH}$ end group of HCQ is of the appropriate length to extend across and attach through the oxygen nucleophile by ionic coupling to the phosphate backbone of the adenine nucleotide, in a manner like steroid hormones. Moreover, the two aromatic rings of HCQ, provide structural stability to enable the simultaneous attachment of the $\mathrm{Cl}$ element and the $\mathrm{NH}$ group to the Uracil nucleotide. Based on my earlier work in discovering the structural basis of DNA as the steroid molecule, and its relation to steroid hormones, $[16,17]$ these requirements are essential for binding to DNA nucleotides, and thus by extension, essential for binding to RNA nucleotides.

Moreover, the structural biology analysis of azithromycin (AZR) is in excellent agreement with the hypothesis put forth in this research article that AZR functions as a receptor for HCQ to assemble and coordinate the HCQ molecule with two positively charged ions and then deliver and release HCQ, along with the two ions, to bind the AU nucleotide pairing, and thus inhibit the replication of the coronavirus disease COVID-19. Like HCQ, AZR has an exceptional molecular structure to enable its contribution to the defeat of the coronavirus disease COVID-19. As the function of AZR with HCQ is as its receptor and delivery vector, AZR has a small molecular structure, which will permit a significant rate of intracellular mobility in comparison to the glucocorticoid receptor. It is of a size that is compatible with the HCQ molecule, and to hold and separate two positively charged ions, which can be $\mathrm{Mg}^{2+}, \mathrm{Zn}^{2+}, \mathrm{Ca}^{2+}$. Moreover, one side is completely open, which permits the two aromatic rings of $\mathrm{HCQ}$, along with its $\mathrm{Cl}$ end group, to couple with AZR. Furthermore, the open region extends to the NH group of HCQ, such that, in combination with the $\mathrm{Cl}$ end group of HCQ, a clear path is established to enable a coupling with the Uracil nucleotide at adjacent phosphate groups and at the available oxygen-based functional group of Uracil. In addition, AZR has a group that partially and temporarily blocks the $\mathrm{OH}$ end group of HCQ from interacting with the AU nucleotide, until HCQ completes its binding to the Uracil nucleotide. Moreover, the positioning of the second ion element permits its rotation toward the adenine nucleotide to enable the completion of the binding of the RNA strands, configured as a secondary structure, so as to inhibit the coronavirus disease.

Thus, a mechanism of action for HCQ and AZR is developed for affecting and inhibiting the translation and replication processes of the coronavirus disease COVID-19. From this mechanism of action, insight into the proper use of HCQ and AZR is gained such that proper medical decisions can be made, which includes: (1) HCQ is more effective than chloroquine (CQ), which does not have an $\mathrm{OH}$ end group, and thus, while CQ can effectively bond with the Uracil nucleotide, it will have difficulty bonding to the Adenine nucleotide, since only the nitrogen group is available, which is poorly positioned and is not as strong a nucleophile as the $\mathrm{OH}$ end group of HCQ; (2) HCQ can work moderately without AZR, because its assembly with two ions and its transport to the AU nucleotide pairing can be accomplished with the glucocorticoid receptor, or can be constructed independently of a receptor; (3) HCQ with AZR is the most effective combination to inhibit the coronavirus 
disease, because it combines a relatively small receptor as a coupling and transport agent to deliver HCQ as a stabilization molecule in which there is exceptional agreement with the structural configuration of the AU nucleotide pairings of secondary arrangements of RNA single strand diseases; (4) The coupling agents $\mathrm{Mg}^{2+}, \mathrm{Zn}^{2+}$, or $\mathrm{Ca}^{2+}$, are critical to the coordination of HCQ with AZR, and to its ultimate coupling with AU, and thus supplementation to promote this coupling is appropriate.

With this mechanism of action defined, it is possible to determine how to improve upon the effectiveness of the cross-coupling agent HCQ and its delivery molecule AZR. The strongest coupling will be the steroid molecule of the $\mathbb{H}$ class, such as cortisol, which can strongly bind the RNA strands together, because of its additional rings in addition to a more nucleophilic end group, and thus from this perspective the use of corticosteroids is appropriate (however there are side effects to consider which will be discussed in the following paragraph), which would utilize the glucocorticoid receptor as its delivery mechanism. If HCQ is utilized as the RNA binding agent, it seems that AZR is a near perfect delivery vesicle, and will be difficult to improve; however, if adjustments are made to HCQ, such as improving the nucleophilic arrangement of the end group to enhance stability, then an alternative receptor molecule can be considered in tandem. It is conceivable for HCQ to couple to the AU nucleotide pairings without a receptor vehicle, although it would require that the ionic coupling mechanisms are first in place along the RNA strands, and then would require that there is alignment and attachment to Uracil of both the $\mathrm{Cl}$ element and the NH group; and ultimately the side chain of HCQ with the $\mathrm{OH}$ end group would need to be directed toward an ion element residing within the phosphate groups associated with adenine.

In addition, with this mechanism of action, the side effects of HCQ and AZR can be evaluated. Since HCQ is compatible with occupying the glucocorticoid receptor (GR), side effects may occur as it will interfere with the normative processing of steroid hormones similar to cortisol. On the topic of contaminant to the GR, I have written on it in terms of prostaglandin E2, which can be used for guidance [16, 18]. This interference with the GR may limit the dosing to HCQ. The side effects can be reduced if the HCQ molecule is altered such that it is not compatible with the glucocorticoid receptor, which is challenging because in order for its use as an RNA coupling agent, it should exhibit behavior similar to the target of the glucocorticoid receptor, cortisol. The receptor molecule AZR will not interfere with the normative processing of cortisol, and thus higher dosing of AZR could be considered as it would seem to be beneficial in terms of increasing HCQ processing while decreasing GR-contaminant related side effects causing misprocessing of cortisol.

Relating to the integration at the systems level to assess side effects, this interaction of HCQ with RNA nucleotides supports the discovery of the structural basis of DNA as the steroid molecule [16], in which the purine and pyrimidine bases form three of the four rings of the steroid molecule, and their coordination form the fourth ring through hydrogen bonding; and this work supports the insight of the structural relation of DNA to steroid hormones, which involves a coupling through the construction of a hydrogen bond between 
Thymine and the corticosteroid class of hormones similar in structure to cortisol $[16,17]$. As seen for the RNA strands of the coronavirus configured in a secondary structure involving intramolecular loops to form an A-U pair, the mapping of HCQ to Uracil is coordinated in a complementary manner to that of cortisol to Thymine in DNA nucleotides. Thus, these results are consistent with an integration into an overall theory at the cellular and systems levels, which map misprocessing of steroid hormones as causative for certain signs and symptoms of disease, in particular fever and fatigue. Therefore, this analysis strategy can be used collectively to optimize the design of alternatives to HCQ and AZR, or as guidance to determine the proper usage and dosage requirements, and their interaction or supplementation with corticosteroids, to counteract the coronavirus disease COVID-19. These systems level models can thus be used to ascertain the efficacy and the potential side effects.

In general, the technique developed in this article can be applied to evaluate other drug candidates for the inhibition of the replication of SARS-CoV-2 and other single-strand or double-strand RNA viruses. The minimum requirement is to align the structural map of the drug candidate to the RNA nucleotide, particularly the adenine-uracil pair to achieve a combination of three intermolecular and intramolecular hydrogen bonds, along with the capability to achieve binding on each RNA strand. There are other structural considerations as well, such as the angle of the ring structure facing the uracil nucleotide, and the use of a methyl group to act as an intermolecular spacing element. Steroid hormones and steroid molecules are paricularly well suited, but require a better receptor and delivery vehicle, other than the glucocorticoid or androgen receptor. Thus, the description of the requirements for a delivery vesicle, such as that of azithromycin, can also be followed according to the description in this research article. The collection and delivery molecular device require the capability to hold positive two charged ions, and to allow open access to the nucleotide pairs.

These developments thereby indicate excellent structural biological configuration of hydroxychloroquine in relation to the Adenine-Uracil nucleotide pairing of a secondary structure of the single-strand RNA coronavirus, and thus in conjunction with azithromycin as an effective receptor and delivery mechanism, enable a functional treatment process for COVID-19.

\section{Methods}

To construct three dimensional molecular models to evaluate the interaction of steroid hormones and nucleotides, the software program Avogadro was used. The amino acid residues of its ligand binding domain of the glucocorticoid receptor, including the $\alpha$ carbon, were positioned in proximity to the functional groups of hydroxychloroquine or cortisol in accordance with the approximate layout presented in [19], which used crystal studies for identification of the amino acid residues of the ligand binding domain of the glucocorti- 
coid receptor. The force field MMFF94 [20] was utilized and an optimization program available in the software that minimizes the energy associated with the complex was used for determining the bond length and the positioning of the chemical elements, including the functional groups of the ligand binding domain. The dimensions of the spacings were determined at the central location of the carbon, oxygen, hydrogen, nitrogen, calcium, magnesium, zinc, or phosphate element. The software program was run to determine the optimal bond lengths and positioning of the elements. Calcium, magnesium, or zinc ions with a positive two charge were added in the vicinity of negative electrostatic potential and the software program was run to determine the optimal position of the ions. After determining the position, the HCQ molecule and the two associated ions was extracted from the glucocorticoid receptor and placed in the vicinity of the Adenine - Uracil pairing, and the software program was used to determine the optimal locations to minimize the energy. Various initial conditions were evaluated. For the azithromycin results, which included placing two ions within the structure, and then bringing the HCQ molecule in proximity, the methods used were similar to those employed for cortisol and described elsewhere [18]. The description for bringing the HCQ molecule, coupled with Azithromycin, were described in the text.

\section{Bibliography}

[1] D. Baud, X. Qi, K. Nielsen-Saines, D. Musso, L. Pomar, and G. Favre, "Real estimates of mortality following covid-19 infection," The Lancet infectious diseases, 2020.

[2] Y. Roussel, A. Giraud-Gatineau, M.-T. Jimeno et al., "SARS-CoV-2: fear versus data," International Journal of Antimocrobial Agents, 2020, https://doi.org/10.1016/j.ijantimicag.2020.105947.

[3] A. Miller, M. J. Reandelar, K. Fasciglione, V. Roumenova, Y. Li, and G. H. Otazu, "Correlation between universal bcg vaccination policy and reduced morbidity and mortality for covid-19: an epidemiological study," medRxiv, 2020.

[4] K. Dhama, K. Sharun, R. Tiwari, M. Dadar, Y. S. Malik, K. P. Singh, and W. Chaicumpa, "Covid-19, an emerging coronavirus infection: advances and prospects in designing and developing vaccines, immunotherapeutics, and therapeutics," Human Vaccines \& Immunotherapeutics, pp. 1-7, 2020.

[5] P. Gautret, J. Lagier, P. Parola, V. Hoang, L. Meddeb, M. Mailhe, B. Doudier, J. Courjon, V. Giordanengo, V. Vieira, H. Dupont, S. Honoré, P. Colson, E. Chabrière, B. La Scola, J. Rolain, P. Brouqui, and D. Raoult, "Hydroxychloroquine and azithromycin as a treatment of COVID-19: results of an open-label non-randomized clinical trial," International Journal of Antimicrobial Agents, 2020, https://doi.org/10.1016/j.ijantimicag.2020.105949. 
[6] J. Liu, R. Cao, M. Xu, X. Wang, H. Zhang, H. Hu, Y. Li, Z. Hu, W. Zhong, and M. Wang, "Hydroxychloroquine, a less toxic derivative of chloroquine, is effective in inhibiting sars-cov-2 infection in vitro," Cell discovery, vol. 6, no. 1, pp. 1-4, 2020.

[7] D. Zhou, S.-M. Dai, and Q. Tong, "Covid-19: a recommendation to examine the effect of hydroxychloroquine in preventing infection and progression," Journal of Antimicrobial Chemotherapy, 2020.

[8] M. Gendrot, E. Javelle, E. Le Dault, A. Clerc, H. Savini, and B. Pradines, "Chloroquine as prophylactic agent against COVID-19?" International Journal of Antimicrobial Agents, 2020, https://doi.org/10.1016/j.ijantimicag.2020.105980.

[9] E. Cohen and M. Nigam, "President Trump is wrong in so many ways about hydroxychloroquine studies. Here are the facts," 2020, posted on-line https://www.cnn.com/2020/04/10/health/trump-wrong-abouthydroxychloroquine/index.html.

[10] S. S. Hasan, C. S. Kow, and H. Merchant, "Is it worth the wait? should chloroquine or hydroxychloroquine be allowed for immediate use in covid-19?" British Journal of Pharmacy, vol. 5, no. 1, p. 745, 2020.

[11] S. D'Alessandro, D. Scaccabarozzi, L. Signorini, F. Perego, D. Ilboudo, P. Ferrange, and S. Delbue, "The Use of Antimalarial Drugs against Viral Infection," Microorganisms, vol. 8, 2020, https://doi.org/10.3390/microorganisms8010085.

[12] P. Colson, J.-M. Rolain, J. C. Lagier, P. Brouqui, and D. Raoult, "Chloroquine and hydroxychloroquine as available weapons to fight covid-19," International Journal of Antimicrobial Agents, 2020, https://doi.org/10.1016/j.ijantimicag.2020.105932.

[13] E. Schrezenmeier and T. Dorner, "Mechanisms of action of hydroxychloroquine and chloroquine: implications for rheumatology," Nature Reviews Rheumatology, vol. 16, pp. 155-166, 2020.

[14] J. Fantini, C. Di Scala, H. Chahinian, and N. Yahi, "Structural and molecular modeling studies reveal a new mechanism of action of chloroquine and hydroxychloroquine against sars-cov-2 infection," International Journal of Antimicrobial Agents, 2020, https://doi.org/10.1016/j.ijantimicag.2020.105960.

[15] X. Yao et al., "In vitro antiviral activity and projection of optimized dosing design of hydroxychloroquine for the treatment of severe acute respiratory syndrome coronavirus 2 (sars-cov-2)," Clinical Infectious Diseases, 2020, https://doi.org/10.1093/cid/ciaa237. 
[16] C. Schaper, "Endogenous Binding of Steroid Molecules to DNA Nucleotides by a Ca2+/PO4- Process to Enable Gene Transcription," ChemRxiv, 2020, https://doi.org/10.26434/chemrxiv.11868261.v2.

[17] — - "Structural Symmetry of DNA Nucleotides and Steroid Hormones," ChemRxiv, 2020, https://doi.org/10.26434/chemrxiv.11991567.v1.

[18] — - "Competitive Inhibition of Cortisol by Prostaglandins at the Ligand Binding Domain of Glucocorticoid Receptors," bioRxiv, 2019, https://doi.org/10.1101/851501.

[19] X. Liu, Y. Wang, and E. A. Ortlund, "First high-resolution crystal structures of the glucocorticoid receptor ligand-binding domain-peroxisome proliferator-activated $\gamma$ coactivator 1- $\alpha$ complex with endogenous and synthetic glucocorticoids," Molecular Pharmacology, vol. 96, no. 4, pp. 408-417, 2019.

[20] T. A. Halgren, "Merck molecular force field. i. basis, form, scope, parameterization, and performance of mmff94," Journal of computational chemistry, vol. 17, no. 5-6, pp. 490-519, 1996. 